\title{
A GENERALIZED KOSZUL COMPLEX. III. A REMARK ON GENERIC ACYCLICITY
}

\author{
DAVID A. BUCHSBAUM AND DOCK SANG RIM ${ }^{1}$
}

1. Introduction. Let $R$ be a commutative ring, and $S=R\left[X_{11}, \cdots\right.$, $X_{m n}$ ] be the polynomial ring in $m n$ indeterminates over $R$. If we denote by $g(m, n): S^{m} \rightarrow S^{n}$ the map whose matrix is $\left(X_{i j}\right)$, we may consider the (generalized) Koszul complexes $K\left(\bigwedge^{p} g(m, n)\right)$ for $1 \leqq p \leqq n$ [2], [3]. One of the first things we shall show is that these complexes are acyclic, i.e. $H_{i}\left(K\left(\Lambda^{p} g(m, n)\right)\right)=0$ for $i>0$. We will do this by using a result in a recent paper by Northcott [4], which says that if $R$ is noetherian, then the ideal, $I$, generated by the subdeterminants of order $n$ of the matrix $\left(X_{i j}\right)$ is perfect, and $\operatorname{depth}(I ; S)=m-n+1$.

Because the complexes $K\left(\Lambda^{p} g(m, n)\right)$ are acyclic, we say that the generalized Koszul complex is generically acyclic. This generic acyclicity enables us to interpret the homology and cohomology groups of the generalized Koszul complex as Tor and Ext over suitable rings, and also expresses these groups as the homology (and cohomology) of the classical Koszul complex. As a result, we are able to give easy proofs of the rigidity of these complexes [1], and also prove an assertion [2] concerning the annihilator of their homology groups.

Throughout this note, all rings are commutative and have an identity element.

2. Generic acyclicity. As in $\S 1$, we let $S=R\left[X_{11}, \cdots, X_{m n}\right]$, where $X_{i j}$ are indeterminates over $R, 1 \leqq i \leqq m, 1 \leqq j \leqq n(m \geqq n)$, and $g(m, n): S^{m} \rightarrow S^{n}$ is the map whose matrix is $\left(X_{i j}\right)$. The ideal $I(m, n)$ (or $I$ ) generated by the subdeterminants of $\left(X_{i j}\right)$ of order $n$ is simply the image of the map $\Lambda^{n} g(m, n): \Lambda^{n} S^{m} \rightarrow \Lambda^{n} S^{n}$.

TheOREM 2.1. For each $p(1 \leqq p \leqq n)$, the complex $K\left(\bigwedge^{p} g(m, n)\right)$ $[3, \S 1]$ has trivial homology in positive dimensions. Thus $K\left(\Lambda^{p} g(m, n)\right)$ is a free resolution of Coker $\bigwedge^{p} g(m, n)$.

Proof. We know, by Northcott's result quoted in $\$ 1$, that, in case $R$ is noetherian, we have $\operatorname{depth}(I ; S)=m-n+1[3, \S 2]$. Thus, if $R$ is noetherian, the theorem follows immediately from Corollary 2.6 of [3]. In case $R$ is not noetherian, we observe that $R=\lim _{\rightarrow} R_{\alpha}$ where $R_{\alpha}$ is noetherian, and hence $S=\lim _{\rightarrow} S_{\alpha}$, where $S_{\alpha}=R_{\alpha}\left[X_{11}, \cdots, X_{m n}\right]$.

Received by the editors January 20, 1964.

1 This work was done with the partial support of NSF grant GP-218. 
Therefore, $K\left(\Lambda^{p}(g(m, n), S)=\lim _{\rightarrow} K\left(\Lambda^{p} g(m, n), S_{\alpha}\right)\right.$ and we see that $K\left(\Lambda^{p} g(m, n), S\right)$ is acyclic.

CoRollaRy 2.2. Given a map $f: R^{m} \rightarrow R^{n}$ with matrix $\left(a_{i j}\right)$ and $a$ finitely generated $R$-module $E$, we have

$$
\begin{aligned}
& H_{*}\left(\Lambda^{p} f, E\right)=\operatorname{Tor}^{S}\left(\operatorname{Coker} \Lambda^{p} g(m, n), \bar{S} \otimes_{R} E\right), \\
& H^{*}\left(\Lambda^{p} f, E\right)=\operatorname{Ext}_{S}\left(\operatorname{Coker} \Lambda^{p} g(m, n), \operatorname{Hom}_{R}(\bar{S}, E)\right),
\end{aligned}
$$

where $S=R\left[X_{11}, \cdots, X_{m n}\right]$ and $\bar{S}=S / I(X(f))$, with $I(X(f))$ $=\left(X_{11}-a_{11}, \cdots, X_{m n}-a_{m n}\right)$. (For definitions of $H_{*}$ and $H^{*}$, see [3, \$1].)

Proof. This follows immediately from the fact that $K\left(\Lambda^{p} g(m, n)\right)$ is a free resolution of Coker $\Lambda^{p} g(m, n)$ and that $K\left(\Lambda^{p} f\right) \otimes_{R} E$ $=\left(K\left(\bigwedge^{p} g(m, n)\right) \otimes_{S} \bar{S}\right) \otimes_{R} E=K\left(\bigwedge^{p} g(m, n)\right) \otimes_{S}\left(\bar{S} \otimes_{R} E\right)$ and

$$
\begin{aligned}
\operatorname{Hom}_{R}\left(K\left(\Lambda^{p} f\right), E\right) & =\operatorname{Hom}_{R}\left(K\left(\Lambda^{p} g(m, n)\right) \otimes_{S} \bar{S}, E\right) \\
& =\operatorname{Hom}_{S}\left(K\left(\Lambda^{p} g(m, n)\right), \operatorname{Hom}_{R}(\bar{S}, E)\right) .
\end{aligned}
$$

Corollary 2.3. $H\left(\Lambda^{p} f, \cdot\right)$ is annihilated by Ann Coker $\Lambda^{p} f(c f$. [2]).

As another application we obtain the rigidity of the complex $K\left(\bigwedge^{p} f\right)$.

ThEOREM 2.4. Let $f$ and $S$ be as in 2.2, with $R$ noetherian. If $X(f)$ : $S^{m n} \rightarrow S$ is the map whose $m n \times 1$ matrix is $\left(X_{11}-a_{11}, \cdots, X_{m n}-a_{m n}\right)$, then $H_{*}\left(\bigwedge^{p} f, E\right)=H_{*}\left(X(f), E \otimes_{R}\right.$ Coker $\left.\bigwedge^{p} g(m, n)\right)$. In particular, $H_{s}\left(\bigwedge^{p} f, E\right)=0$ implies $H_{t}\left(\bigwedge^{p} f, E\right)=0$ for all $t \geqq s$.

Proof. $K\left(\bigwedge^{p} g(m, n)\right)$ is an acyclic resolution of Coker $\bigwedge^{p} g(m, n)$, regardless of the coefficient ring $R$. In particular, this is so for $R / \mathfrak{a}$ for any ideal $a$ in $R$, i.e., $K\left(\wedge^{p} g(m, n)\right) \otimes_{R} R / \mathfrak{a}$ is acyclic for any ideal a. Thus $\operatorname{Tor}^{R}\left(\right.$ Coker $\left.\Lambda^{p} g(m, n), R / \mathfrak{a}\right)=0$ for all ideals $\mathfrak{a}$, since $K\left(\Lambda^{p} g(m, n)\right)$ is $S$-free and, hence, an $R$-free resolution of Coker $\Lambda^{p} g(m, n)$. Therefore Coker $\Lambda^{p} g(m, n)$ is flat as an $R$-module.

Now the standard Koszul complex $K(X(f))$ is an $S$-free resolution of Coker $X(f)=\bar{S}$, since $I(X(f))$ is generated by an $S$-sequence (as can be seen by linear substitution). Since $\bar{S}=R$ as an $R$-module, 
$K(X(f)) \otimes_{R} E$ is an acyclic resolution of $\bar{S} \otimes_{R} E$, and each term of $K(X(f)) \otimes_{R} E$ is a direct sum of copies of $S \otimes_{R} E$. Now

$\operatorname{Tor}_{i}^{S}\left(\operatorname{Coker} \Lambda^{p} g(m, n), S \otimes_{R} E\right)=\operatorname{Tor}_{i}^{R}\left(\operatorname{Coker} \Lambda^{p} g(m, n), E\right)=0$,

for all $i>0$, since Coker $\Lambda^{p} g(m, n)$ is $R$-flat. Therefore,

$$
\begin{aligned}
& H_{*}\left(X(f), E \otimes_{R} \operatorname{Coker} \stackrel{p}{\Lambda}_{g(m, n)}\right) \\
& =H_{*}\left(K(X(f)) \otimes_{S}\left(E \otimes_{R} \operatorname{Coker} \stackrel{p}{\Lambda}_{g}(m, n)\right)\right) \\
& =H_{*}\left(\operatorname{Coker} \stackrel{p}{\Lambda}_{g}(m, n) \otimes_{S}\left(K(X(f)) \otimes_{R} E\right)\right) \\
& =\operatorname{Tor}^{S}\left(\operatorname{Coker} \Lambda^{p} g(m, n), \bar{S} \otimes_{R} E\right)=H_{*}\left(\bigwedge^{p} f, E\right) \cdot{ }^{2}
\end{aligned}
$$

Corollary 2.5. Let $R$ be noetherian, and $f, S$ be as above. Then $\operatorname{depth}(I(f) ; R)=m-n+1$ if and only if $\operatorname{depth}(I(X(f)) ; S / I(g(m, n)))$ $=m n$. If $R$ is a local ring and every entry $a_{i j}$ of the matrix of $f$ is contained in the maximal ideal, $\mathfrak{m}$, of $R$ (i.e., $f\left(R^{m}\right) \subset \mathfrak{m} R^{n}$ ), then depth $(I(f) ; R)=m-n+1$ if and only if $\left\{X_{11}-a_{11}, \cdots, X_{m n}-a_{m n}\right\}$ is an $(S / I(g(m, n)))_{\tilde{\mathfrak{m}}}$-sequence, where $\tilde{\mathfrak{m}}=\left(\mathfrak{m}, X_{11}, \cdots, X_{m n}\right)$.

Proof. The first statement follows from the above, namely $H_{*}\left(\bigwedge^{n} f, R\right)=H_{*}(X(f), S / I(g(m, n))$ ) (letting $p=n)$.

Now assume that $R$ is local and all the $a_{i j}$ are in $\mathrm{m}$. Then

$$
H_{i}(X(f), S / I(g(m, n)))=0
$$

if and only if $H_{i}(X(f), S / I(g(m, n)))_{\tilde{\mathfrak{m}}}=0$ since $\tilde{\mathfrak{m}}$ is the unique maximal ideal of $S$ containing $I(X(f))$. Since $\left(X_{11}-a_{11}, \cdots, X_{m n}-a_{m n}\right)$ is contained in $\tilde{\mathfrak{m}}, H_{*}(X(f), S / I(g(m, n)))=0$ for all positive dimensions if and only if $X_{11}-a_{11}, \cdots, X_{m n}-a_{m n}$ is an $(S / I(g(m, n)))_{\tilde{m}^{-}}$sequence.

Let $R$ be a local ring, $M$ an $R$-module of finite length, and $R^{m} \rightarrow R^{n}$ $\rightarrow M \rightarrow 0$ an exact sequence. We have shown in $[3,3.4]$ that $P_{f}(\nu, R)$ $=l\left(\right.$ Coker $\left.S_{v}(f)\right)$ is a polynomial function whose degree is $n-1$

2 The next to the last equality is obtained by using the following well-known fact: if $S$ is a ring, $Y=\left\{Y_{n}\right\}$ an acyclic complex over the $S$-module $B$, and $A$ an $S$-module such that $\operatorname{Tor}_{i}{ }^{S}\left(A, Y_{n}\right)=0$ for all $i>0$ and all $n$, then $\operatorname{Tor}_{i}{ }^{S}(A, B)=H_{i}\left(A \otimes_{S} Y\right)$. 
$+\operatorname{dim} R$, and that $e_{R}(M)=(n-1+\operatorname{dim} R)$ ! (leading coefficient of $P_{f}(\nu, R)$ ) is a positive integer which depends only on the module $M$. Furthermore, we have shown $[3,4.3]$ that

$$
\chi H_{*}\left(\stackrel{p}{\Lambda}_{f}, R\right)=\left(\begin{array}{l}
n-1 \\
n-p
\end{array}\right) e_{R}(M)
$$

if $f$ is a parameter matrix, i.e., if $m-n+1=\operatorname{dim} R$, where $\chi H_{*}$ stands for the Euler-Poincaré characteristic. We observe that $f$ is a parameter matrix if and only if $I(X(f))$ is an ideal of definition for $\tilde{S}$ generated by a system of parameters, where $S=R\left[X_{11}, \cdots, X_{m n}\right]$ and $\tilde{S}=(S / I(g(m, n)))_{\tilde{m}}$. Therefore, Theorem 2.4 yields immediately

COROLlaRY 2.6. Let $f: R^{m} \rightarrow R^{n}$ be a parameter matrix. Then $e_{R}(M)$ $=e_{\tilde{S}}(\tilde{S} / I(X(f)))$, where $M=$ Coker $f$.

\section{BIBLIOGRAPHY}

1. M. Auslander, Modules over unramified regular local rings, Illinois J. Math. 5 (1961), 631-647.

2. D. Buchsbaum, A generalized Koszul complex. I, Trans. Amer. Math. Soc. 111 (1964), 183-196.

3. D. Buchsbaum and D. S. Rim, A generalized Koszul complex. II. Depth and multiplicity, Trans. Amer. Math. Soc., 111 (1964), 197-224.

4. D. G. Northcott, Some remarks on the theory of ideals defined by matrices, Quart. J. Math. Oxford Ser. (2) 14 (1963), 193-204.

BRANDEIS UNIVERSITY

\section{ERRATA, VOLUME 16}

Culbreth Sudler, Jr., A direct proof of two theorems on two-line partitions, pp. 161-168.

Page 165, (3.2). For $a_{\nu}$ read $\alpha_{\nu}$.

Page 167, line -1. For 9531 , read $\begin{array}{llll}11 & 5 & 1 .\end{array}$ 